\title{
An Experimental Model of Tennis Elbow in Rats: A Study of the Contribution of the Nervous System
}

\author{
Eva Haker, Elvar Theodorsson, and Thomas Lundeberg
}

Erratum to: Inflammation (1998) 22:435-444

DOI 10.1023/A:1022377001084

The Editors of Inflammation retracted this article due to a finding of plagiarism and possible scientific fraud on the part of Dr. Lundeberg following an investigation at the Karolinska Institutet which was completed in 2007.

The online version of the original article can be found at http://dx.doi. org/10.1023/A:1022377001084 